\title{
Dirac spectrum representation of Polyakov loop fluctuations in lattice QCD *
}

\author{
Takahiro M. Doi ${ }^{\dagger}$ \\ Department of Physics, Kyoto University, Kyoto 606-8502, Japan \\ E-mail: doi@ruby.scphys.kyoto-u.ac.jp
}

\section{Krzysztof Redlich}

Institute of Theoretical Physics, University of Wroclaw, PL-50204 Wroclaw, Poland Extreme Matter Institute EMMI, GSI, Planckstr. 1, D-64291 Darmstadt, Germany

Department of Physics, Duke University, Durham, North Carolina 27708, USA

\section{Chihiro Sasaki}

Institute of Theoretical Physics, University of Wroclaw, PL-50204 Wroclaw, Poland

Frankfurt Institute for Advanced Studies, D-60438 Frankfurt am Main, Germany

\section{Hideo Suganuma}

Department of Physics, Kyoto University, Kyoto 606-8502, Japan

\begin{abstract}
Dirac spectrum representations of the Polyakov loop fluctuations are derived on the temporally odd-number lattice, where the temporal length is odd with the periodic boundary condition. We investigate the Polyakov loop fluctuations based on these analytical relations. It is semianalytically and numerically found that the low-lying Dirac eigenmodes have little contribution to the Polyakov loop fluctuations, which are sensitive probe for the quark deconfinement. Our results suggest no direct one-to-one corresponding between quark confinement and chiral symmetry breaking in QCD.
\end{abstract}

The 33rd International Symposium on Lattice Field Theory

14 - 18 July 2015

Kobe International Conference Center, Kobe, Japan*

${ }^{*}$ T. M. D. is supported by Grant-in-Aid for JSPS Fellows (Grant No. 15J02108), and H. S. is supported by the Grants-in-Aid for Scientific Research (Grant No. 15K05076) from Japan Society for the Promotion of Science. K. R. acknowledges partial support of the U.S. Department of Energy under Grant No. DE-FG02- 05ER41367, and fruitful discussions with Bengt Friman and Pok Man Lo. The work of K. R. and C. S. has been partly supported by the Polish Science Foundation (NCN) under Maestro Grant No. DEC-2013/10/A/ST2/00106 and by the Hessian LOEWE initiative through the Helmholtz International Center for FAIR. The lattice QCD calcula- tions were performed on NEC-SX8R and NEC-SX9 at Osaka University.

† Speaker. 


\section{Introduction}

It is one of the most important problems in particle and nuclear physics to understand the nonperturbative properties of QCD, such as confinement and chiral symmetry breaking, including these relation. By numerous efforts, these properties have been partially understood.

The Polyakov loop is an order parameter of quark confinement in the quenched QCD [1]. Moreover, recently it is pointed out that the Polyakov loop fluctuations are very sensitive probes for the quark deconfinement even in full QCD with light quarks [2].

The low-lying eigenmodes of the Dirac operator are important for chiral symmetry breaking, for example known as Banks-Casher relation [3]. At low temperature, the low-lying Dirac modes exist and have dominant contribution to chiral condensate while at high temperature these modes disappear and chiral symmetry is restored.

In the presence of light dynamical quarks, both quark deconfinement and chiral restoration are not phase transition but crossover and take place in the same temperature region [4, 5, 6, 7]. This observation seems evidence that confinement and chiral symmetry breaking are strongly correlated in QCD. However, there are some contrary observations that chiral restoration does not immediately mean the quark deconfinement. For example, "hadrons" can be observed as bound states after removal of low-lying Dirac modes [8]. Also, it is shown that low-lying Dirac modes are not important for confinement properties such as the Polyakov loop and the linear confining potential of a quark-antiquark system 9 .

In this paper, we derive some analytical relations to express the Polyakov loop and its fluctuations by Dirac eigenmodes. Then we semi-analytically and numerically show that low-lying Dirac modes have negligible contribution to the Polyakov loop fluctuations based on these analytical relations. This talk is mainly based on our recent work [10].

\section{Polyakov loop fluctuations and Dirac mode on a lattice}

In this section, we review the Polyakov loop fluctuations [2] and Dirac-mode expansion [9] as the basics of our work. In this paper, we consider the $\mathrm{SU}\left(N_{\mathrm{c}}\right)$ lattice QCD formalism on a square lattice with spacing $a$. We denote each site as $s=\left(s_{1}, s_{2}, s_{3}, s_{4}\right)$ with $s_{\mu}=1,2, \cdots, N_{\mu}$ and a link variable as $U_{\mu}(s)=\mathrm{e}^{i a g A_{\mu}(s)}$ with a gauge field, $A_{\mu}(s) \in s u\left(N_{c}\right)$, and the gauge coupling $g$. We use spatially symmetric lattice, i.e., $N_{1}=N_{2}=N_{3} \equiv N_{\sigma}$ and $N_{4} \equiv N_{\tau}$, with $N_{\sigma} \geq N_{\tau}$. We define all the $\gamma$-matrices to be hermite as $\gamma_{\mu}^{\dagger}=\gamma_{\mu}$.

\subsection{Polyakov loop fluctuations}

For each gauge configuration, the Polyakov loop $L$ and the $Z_{3}$ rotated Polyakov loop $\tilde{L}$ are defined as

$$
L \equiv \frac{1}{N_{\mathrm{c}} V} \sum_{s} \operatorname{tr}_{c}\left\{\prod_{i=0}^{N_{\tau}-1} U_{4}(s+i \hat{4})\right\}, \quad \tilde{L} \equiv L \mathrm{e}^{2 \pi k i / 3} \quad(k=0, \pm 1)
$$

where $\hat{\mu}$ is the unit vector in direction of $\mu$ in the lattice unit, $V=N_{\sigma}^{3} N_{\tau}$ is the volume of 4dimensional lattice, $k=0$ is taken in the confinement phase, and $k$ in the deconfinement phase 
is chosen such that the transformed Polyakov loop $\tilde{L}$ lies in its real sector. Using the $Z_{3}$ rotated Polyakov loop $\tilde{L}$, three Polyakov loop susceptibilities are introduced as

$$
\begin{aligned}
T^{3} \chi_{A} & =\frac{N_{\sigma}^{3}}{N_{\tau}^{3}}\left[\left\langle|L|^{2}\right\rangle-\langle|L|\rangle^{2}\right], \\
T^{3} \chi_{L} & =\frac{N_{\sigma}^{3}}{N_{\tau}^{3}}\left[\left\langle\left(L_{L}\right)^{2}\right\rangle-\left\langle L_{L}\right\rangle^{2}\right], \\
T^{3} \chi_{T} & =\frac{N_{\sigma}^{3}}{N_{\tau}^{3}}\left[\left\langle\left(L_{T}\right)^{2}\right\rangle-\left\langle L_{T}\right\rangle^{2}\right],
\end{aligned}
$$

where $L_{L} \equiv \operatorname{Re}(\tilde{L})$ and $L_{T} \equiv \operatorname{Im}(\tilde{L})$, and $\langle x\rangle$ denotes an average over all gauge configurations. Also, their ratios are introduced as,

$$
R_{A} \equiv \frac{\chi_{A}}{\chi_{L}}, \quad R_{T} \equiv \frac{\chi_{T}}{\chi_{L}}
$$

By finite-temperature lattice QCD calculation, it is found that the ratio $R_{A}$ is a very sensitive probe for the quark deconfinement even in the presence of light dynamical quarks [2].

\subsection{Operator formalism and Dirac mode on a lattice}

The link-variable operator $\hat{U}_{ \pm \mu}$ is defined by the matrix element

$$
\left\langle s\left|\hat{U}_{ \pm \mu}\right| s^{\prime}\right\rangle=U_{ \pm \mu}(s) \delta_{s \pm \hat{\mu}, s^{\prime}}
$$

At the temporal boundary, we impose the temporal antiperiodicity to the link-variable operator as

$$
\left\langle\mathbf{s}, N_{t}\left|\hat{U}_{4}\right| \mathbf{s}, 1\right\rangle=-U_{4}\left(\mathbf{s}, N_{t}\right)
$$

to impose the temporal antiperiodicity to the Dirac operator for finite-temperature formalism. Using the link-variable operator, the Polyakov loop is expressed as

$$
L=-\frac{1}{N_{\mathrm{c}} V} \operatorname{Tr}_{c}\left\{\hat{U}_{4}^{N_{\tau}}\right\}=\frac{1}{N_{c} V} \sum_{s} \operatorname{tr}_{c}\left\{\prod_{n=0}^{N_{t}-1} U_{4}(s+n \hat{t})\right\}
$$

where $\operatorname{Tr}_{c}$ denotes the functional trace, $\operatorname{Tr}_{c} \equiv \sum_{s} \operatorname{tr}_{c}$, and $\operatorname{tr}_{c}$ is taken over color index.

Unlike the Polyakov loop, a functional trace of a product of the link-variable operators corresponding to the non-closed path vanishes:

$$
\operatorname{Tr}_{c}\left(\hat{U}_{\mu_{1}} \hat{U}_{\mu_{2}} \cdots \hat{U}_{\mu_{N_{P}}}\right)=\operatorname{tr}_{c} \sum_{s} U_{\mu_{1}}(s) \cdots U_{\mu_{N_{P}}}\left(s+\sum_{k=1}^{N_{P}-1} \hat{\mu}_{k}\right)\left\langle s+\sum_{k=1}^{N_{P}} \hat{\mu}_{k} \mid s\right\rangle=0,
$$

with $\sum_{k=1}^{N_{P}} \hat{\mu}_{k} \neq 0$ for any non-closed path with its length $N_{P}$. This notable property of the linkvariable operator is satisfied due to the definition of the link-variable operator and easily understood by Elitzur' s theorem [11] that a vacuum expectation value of a gauge-variant operator is zero.

The Dirac operator $\hat{\not}$ on the lattice is defined by

$$
\hat{D}=\gamma_{\mu} \hat{D}_{\mu}=\frac{1}{2 a} \sum_{\mu=1}^{4} \gamma_{\mu}\left(\hat{U}_{\mu}-\hat{U}_{-\mu}\right)
$$


The eigenvalue equation of the Dirac operator can be expressed as

$$
\hat{\not D}|n\rangle=i \lambda_{n}|n\rangle, \quad \lambda_{n} \in \mathbf{R}
$$

because of the anti-hermiticity of the Dirac operator. These Dirac eigenstates $|n\rangle$ have the completeness of $\sum_{n}|n\rangle\langle n|=1$. Due to $\left\{\hat{\not}, \gamma_{5}\right\}=0$, the chiral partner $\gamma_{5}|n\rangle$ is also an eigenstate with the eigenvalue $\left(-i \lambda_{n}\right)$. Known as the Banks-Casher relation [3], the low-lying Dirac modes have the dominant contribution to the chiral condensate $\langle\bar{\psi} \psi\rangle$ and thus these modes are essential modes for chiral symmetry breaking.

\section{Dirac spectrum representation of Polyakov loop fluctuations}

In this section, we shortly show the derivation of the Dirac spectrum representations of the Polyakov loop fluctuations and then discuss the relation between confinement and chiral symmetry breaking in QCD. The detailed derivation is shown in Ref. [10,12,13].

We consider a temporally odd-number lattice with odd-number temporal size $N_{\tau}$. On such a lattice, we introduce a functional trace $I$ defined as

$$
I=\operatorname{Tr}_{c, \gamma}\left(\hat{U}_{4} \hat{\not D}^{N_{\tau}-1}\right)
$$

where $\operatorname{Tr}_{c, \gamma} \equiv \sum_{s} \operatorname{tr}_{c} \operatorname{tr}_{\gamma}$, and $\operatorname{tr}_{\gamma}$ is taken over spinor indexes. Substituting the definition of the Dirac operator (2.10), the functional trace $I$ is expressed as a sum of products of odd-number link-variable operators because $N_{\tau}$ is odd number. Note that most of the terms in the expansion of $I$ exactly vanish because one cannot make a closed loop by using odd-number link-variable operators on a square lattice. Thus there is only contribution from the closed path due to the temporal periodicity, that is the Polyakov loop $L$. In this way, the functional trace $I$ can be expressed as

$$
I=\frac{12 V}{(2 a)^{N_{\tau}-1}} L
$$

On the other hand, using the completeness of the Dirac mode, the functional trace is expressed as

$$
I=\sum_{n}\left\langle n\left|\hat{U}_{4} \hat{D}^{N_{\tau}-1}\right| n\right\rangle=i^{N_{\tau}-1} \sum_{n} \lambda_{n}^{N_{\tau}-1}\left\langle n\left|\hat{U}_{4}\right| n\right\rangle
$$

Therefore, we derive the Dirac spectrum representation of the Polyakov loop:

$$
L=\frac{(2 a i)^{N_{\tau}-1}}{12 V} \sum_{n} \lambda_{n}^{N_{\tau}-1}\left\langle n\left|\hat{U}_{4}\right| n\right\rangle .
$$

Since the identity (3.4) is exactly satisfied for each gauge-configuration, the $Z_{3}$ rotated Polyakov loop $\tilde{L}$ can be also expressed by the Dirac modes as

$$
\tilde{L}=\frac{(2 a i)^{N_{\tau}-1}}{12 V} \sum_{n} \lambda_{n}^{N_{\tau}-1} \mathrm{e}^{2 \pi k i / 3}\left\langle n\left|\hat{U}_{4}\right| n\right\rangle,
$$

where $k$ is defined in Eq. 2.1. Correspondingly, we can express $L_{L} \equiv \operatorname{Re}(\tilde{L}), L_{T} \equiv \operatorname{Im}(\tilde{L})$, and $|L|$ by the Dirac modes. From these expressions and Eqs. (2.2)-(2.5), one can derive analytical 
relations connecting the Polyakov loop fluctuations and the Dirac modes. In particular, we focus on the Dirac spectrum representation of $R_{A}$ :

$$
R_{A}=\frac{\left\langle\left|\sum_{n} \lambda_{n}^{N_{\tau}-1}\left\langle n\left|\hat{U}_{4}\right| n\right\rangle\right|^{2}\right\rangle-\left\langle\left|\sum_{n} \lambda_{n}^{N_{\tau}-1}\left\langle n\left|\hat{U}_{4}\right| n\right\rangle\right|\right\rangle^{2}}{\left\langle\left(\sum_{n} \lambda_{n}^{N_{\tau}-1} \operatorname{Re}\left(\mathrm{e}^{2 \pi k i / 3}\left\langle n\left|\hat{U}_{4}\right| n\right\rangle\right)\right)^{2}\right\rangle-\left\langle\sum_{n} \lambda_{n}^{N_{\tau}-1} \operatorname{Re}\left(\mathrm{e}^{2 \pi k i / 3}\left\langle n\left|\hat{U}_{4}\right| n\right\rangle\right)\right\rangle^{2}}
$$

Note that the series of analytical relations is exactly satisfied in both full QCD and quenched QCD.

As mentioned above, the ratio $R_{A}$ is a sensitive probe for the quark deconfinement and the Dirac modes are strongly related to chiral symmtery breaking. Since Eq. (3.6) is an analytical relation connecting the ratio $R_{A}$ and the Dirac modes, we can extract from it the information of the relation between confinement and chiral symmetry breaking in QCD. The damping factors $\lambda_{n}^{N_{\tau}-1}$ in sums over all the Dirac modes appearing in Eq. 3.6 play important roles. Because these damping factors are negligibly small with small eigenvalues $\left|\lambda_{n}\right| \simeq 0$, the contribution from the low-lying Dirac modes to the ratio $R_{A}$ is strongly suppressed while these modes are responsible for saturating the chiral condensate. In other words, important modes for chiral symmetry breaking are not important for chiral symmetry breaking. This analytical discussion is consistent with other numerical studies of the Dirac mode expansion [9], which show that confinement properties such as the Polyakov loop and the confining linear force defined from the Wilson loop are almost unchanged after removal of the low-lying Dirac modes.

Not only the damping factor $\lambda_{n}^{N_{\tau}-1}$, but also the Dirac-mode matrix element $\left\langle n\left|\hat{U}_{4}\right| n\right\rangle$ should be taken into account. If the matrix element increases stronger than the damping factor as $\sim 1 / \lambda_{n}^{N_{\tau}-1}$ in the small- $|\lambda|$ region, the above analytical observation is not correct. However, the matrix element $\left\langle n\left|\hat{U}_{4}\right| n\right\rangle$ does not change our analytical expectation qualitatively. The detailed numerical results of the nontrivial behavior of the matrix element $\left\langle n\left|\hat{U}_{4}\right| n\right\rangle$ are shown in Ref. [13].

\section{Numerical analysis}

In this section, we show the numerical results based on the analytical relation (3.6) to quantitatively confirm the above analytical discussion that the low-lying Dirac modes have negligible contribution to the ratio $R_{A}$.

Since the Dirac eigenvalue $\lambda_{n}$ and the Dirac-mode matrix element $\left\langle n\left|\hat{U}_{4}\right| n\right\rangle$ are gauge-invariant and can be calculated by solving the Dirac eigenvalue equation Eq. 2.11, we can calculate the contribution from each Dirac mode. We introduce the infrared cutoff $\Lambda$ and define the $\Lambda$-dependent Polyakov loops,

$$
|L|_{\Lambda}=\frac{(2 a)^{N_{\tau}-1}}{12 V}\left|\sum_{\left|\lambda_{n}\right|>\Lambda} \lambda_{n}^{N_{\tau}-1}\left\langle n\left|\hat{U}_{4}\right| n\right\rangle\right|
$$

for the modulus, and

$$
\begin{aligned}
\left(L_{L}\right)_{\Lambda} & =C_{\tau} \sum_{\left|\lambda_{n}\right|>\Lambda} \lambda_{n}^{N_{\tau}-1} \operatorname{Re}\left(\mathrm{e}^{2 \pi k i / 3}\left\langle n\left|\hat{U}_{4}\right| n\right\rangle\right) \\
\left(L_{T}\right)_{\Lambda} & =C_{\tau} \sum_{\left|\lambda_{n}\right|>\Lambda} \lambda_{n}^{N_{\tau}-1} \operatorname{Im}\left(\mathrm{e}^{2 \pi k i / 3}\left\langle n\left|\hat{U}_{4}\right| n\right\rangle\right)
\end{aligned}
$$




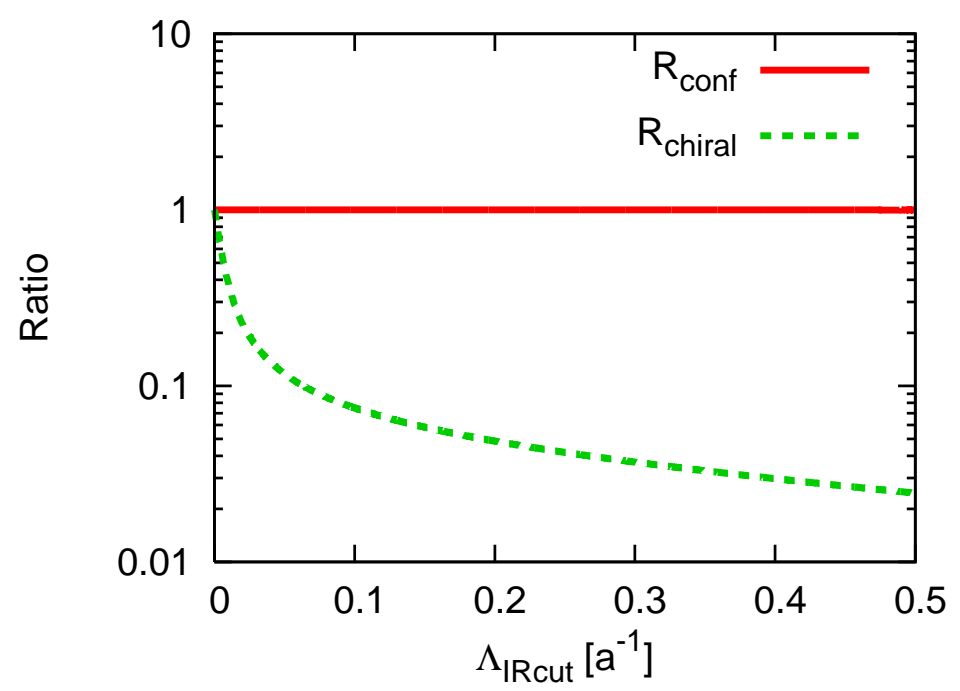

Figure 1: The numerical results for the $R_{\text {chiral }}$ and $R_{\text {conf }}$ plotted against the infrared cutoff $\Lambda$ in lattice units at $\beta=5.6$ on $10^{3} \times 5$ lattice taken from Ref. [10]. The quark mass of $m=5 \mathrm{MeV}$ is taken for calculation of the ratio $R_{\text {chiral }}$.

for the real and the imaginary part, respectively, with $C_{\tau}=(2 a i)^{N_{\tau}-1} / 12 \mathrm{~V}$. Then, the $\Lambda$-dependent Polyakov loop susceptibilities, $\left(\chi_{A}\right)_{\Lambda},\left(\chi_{L}\right)_{\Lambda},\left(\chi_{T}\right)_{\Lambda}$, are defined using the corresponding Polyakov loops, $|L|_{\Lambda},\left(L_{L}\right)_{\Lambda}$ or $\left(L_{T}\right)_{\Lambda}$, and their ratios are also defined as

$$
\left(R_{A}\right)_{\Lambda}=\frac{\left(\chi_{A}\right)_{\Lambda}}{\left(\chi_{L}\right)_{\Lambda}}, \quad\left(R_{T}\right)_{\Lambda}=\frac{\left(\chi_{T}\right)_{\Lambda}}{\left(\chi_{L}\right)_{\Lambda}} .
$$

For comparison, we introduce the cutoff-dependent chiral condensate $\langle\bar{\psi} \psi\rangle_{\Lambda}$ as

$$
\langle\bar{\psi} \psi\rangle_{\Lambda}=-\frac{1}{V} \sum_{\left|\lambda_{n}\right| \geq \Lambda} \frac{2 m}{\lambda_{n}^{2}+m^{2}}
$$

where $m$ is the current quark mass. In order to see the contribution from the low-lying Dirac modes to the ratio $R_{A}$ and chiral condensate, we introduce two quantities $R_{\text {conf }}$ and $R_{\text {chiral }}$ as

$$
R_{\text {conf }}=\frac{\left(R_{A}\right)_{\Lambda}}{R_{A}}, \quad R_{\text {chiral }}=\frac{\langle\bar{\psi} \psi\rangle_{\Lambda}}{\langle\bar{\psi} \psi\rangle} .
$$

The ratios $R_{\text {conf }}$ and $R_{\text {chiral }}$ are calculated in the SU(3) lattice QCD at the quenched level. In our calculation, the standard plaquette action is used on $10^{3} \times 5$ lattice with two different $\beta \equiv \frac{2 N_{\mathrm{c}}}{g^{2}}$ : $\beta=5.6$ for the confinement phase and $\beta=6.0$ for the deconfinement phase. For each $\beta, 20$ gauge-configurations are taken every 500 sweeps after the thermalization of 5000 sweeps.

In Fig. 11, the numerical results for $R_{\text {conf }}$ and $R_{\text {chiral }}$ in the confinement phase are shown with various values of the infrared cutoff $\Lambda$. We take the quark mass $m=5 \mathrm{MeV}$ for the chiral condensate. From Fig. 1, one can confirm that the quantity $R_{\text {chiral }}$ is largely reduced by removal of the low-lying Dirac modes and then the low-lying Dirac modes are important for chiral symmetry breaking. Nevertheless, the quantity $R_{\text {conf }}$, that is the ratio $R_{A}$, is almost unchanged by removing 
the low-lying Dirac modes even with large cutoff $\Lambda \simeq 0.5 \mathrm{GeV}$. The results in the deconfinement phase show the similar behavior with the confinement phase. In this way, we have numerically confirmed that the low-lying Dirac modes have little contribution to the ratio $R_{A}$.

\section{Summary}

We have derived the Dirac spectrum representations of the Polyakov loop and its fluctuations on the temporally odd-number lattice. Based on the analytical relations, it is semi-analytically and numerically shown that the low-lying Dirac modes have negligible contribution to the Polyakov loop fluctuations, which are good probes for the quark deconfinement although these modes have dominant contribution to the chiral condensate. That is, the important modes for chiral symmetry breaking are not important for confinement. Our results suggest no direct one-to-one corresponding between confinement and chiral symmetry breaking in QCD.

\section{References}

[1] H.J. Rothe, "Lattice Gauge Theories", (World Scientific, 2012), and references therein.

[2] P.M. Lo, B. Friman, O. Kaczmarek, K. Redlich and C. Sasaki, Phys. Rev. D88, 014506 (2013); Phys. Rev. D88, 074502 (2013).

[3] T. Banks and A. Casher, Nucl. Phys. B169, 103 (1980).

[4] F. Karsch, Lect. Notes Phys. 583, 209 (2002), and references therein.

[5] F. R. Brown, F. P. Butler, H. Chen, N. H. Christ, Z. Dong, W. Schaffer, Leo I. Unger and Alessandro Vaccarino, Phys. Rev. Lett. 65, 2491 (1990).

[6] Y. Aoki, G. Endrodi, Z. Fodor, S. D. Katz, and K. K. Szabo, Nature (London) 443, 675 (2006).

[7] A. Bazavov et al., Phys. Rev. D85, 054503 (2012).

[8] C.B. Lang and M. Schrock, Phys. Rev. D84, 087704 (2011); L.Ya. Glozman, C.B. Lang, and M. Schrock, Phys. Rev. D86, 014507 (2012).

[9] S. Gongyo, T. Iritani and H. Suganuma, Phys. Rev. D86, 034510 (2012); T. Iritani and H. Suganuma, Prog. Theor. Exp. Phys., 3, 033B03 (2014).

[10] T. M. Doi, K. Redlich, C. Sasaki and H. Suganuma, Phys. Rev. D92, 094004 (2015).

[11] S. Elitzur, Phys. Rev. D12, 3978 (1975).

[12] H. Suganuma, T. M. Doi, T. Iritani, arXiv:1404.6494 [hep-lat]; Proc. Sci. (Lattice 2013), 374 (2013); Proc. Sci. (QCD-TNT-III), 042 (2014); EPJ Web of Conf. 71, 00129 (2014); Proc. Sci. (Hadron 2013), 121 (2014).

[13] T. M. Doi, H. Suganuma, T. Iritani, Phys. Rev. D90, 094505 (2014); Proc. Sci. (Lattice 2013), 375 (2013); Proc. Sci. (Hadron 2013), 122 (2014). 